\title{
A Comparison of Algorithms for Extracting Wind Direction from the Monostatic HF Radar Sea Echoes
}

\author{
Xiaoliang $\mathrm{Chu}^{1,2}$, Jie Zhang ${ }^{2}$ \\ 1.The First Institute of Oceanography, SOA \\ 2. College of Information Science and Engineering, Ocean \\ University of China \\ Qingdao, China
}

\begin{abstract}
In this paper, three algorithms are applied to extract the wind direction using a month HFSWR data in an experiment with a single radar system. The preliminary results show that the three algorithms can all eliminate the ambiguity of the wind direction. And the RMS error (RMSE) obtained by the angle comparison multi-beam (ACMB) algorithm is $24.80^{\circ}$, which is better than the others. We can obtain that the ACMB algorithm is best available for extracting wind direction using the data obtained by this experiment. However, for the other data with different conditions, whether this algorithm is the best needs further examination.
\end{abstract}

Keywords-High frequency surface wave radar (HFSWR), wind direction, ambiguity elimination

\section{Introduction}

High frequency surface wave radar (HFSWR) can be used to track the moving targets and monitor the ocean surface parameters such as currents, wave and wind field. Both theory simulations and experiments confirm that the ratio of spectral power density of the positive and negative Bragg peaks is highly sensitive to the wind direction and so this can be used to extract wind direction [1-4]. However, for the monostatic radar, there exists a directional ambiguity as the radar cannot tell if the wind is from the right of the radar beam or the left. To solve this problem, some methods are developed [2-5]. Heron \& Rose [2] presented a multi-beam (MB) method which finds spreading parameter and wind directions from three radar beams. Wyatt [3] applied a maximum likelihood (ML) method to estimate short wave directions and spreading. These methods can solve the problem of wind direction ambiguity for the monstatic radar. Based on the work of Heron \& Rose, we have developed an algorithm to eliminate the ambiguity which is applied by comparing the included angles between three adjacent radar beams and the wind direction [6], which can be called angle comparison multi-beam( ACMB) in here. And we improve on the multi-beam algorithm by using the least square principle to increase efficiency of calculation (LSMB). In this paper we evaluate the three algorithms ACMB, LSMB, ML, using a month data obtained in an experiment by means of a monostatic radar system, and the accuracy of wind direction measurements are explored.

\author{
Shuyao Wang ${ }^{3}$, Yonggang $\mathrm{Ji}^{1}$, Yiming Wang ${ }^{1}$ \\ ${ }^{3}$ CSIC PRIDE (NanJing) Atmospheric and Oceanic \\ Information System Co.Ltd \\ Nanjing, China \\ xlchu@ouc.edu.cn
}

\section{Brief Description of Algorithm to Extract Wind Direction}

The ratio of spectral power density of two Bragg peaks has been previously used to estimate the wind direction, the firstorder cross section is given by Barrick [7]

$$
\sigma_{1}(\omega)=2^{6} \pi k_{0}^{4} \sum_{m= \pm 1} S\left(-2 m \vec{k}_{0}\right) \delta\left(\omega-m \omega_{\mathrm{B}}\right)
$$

where $\omega$ is the Doppler frequency, $\omega_{B}$ is the first-order Bragg frequency, $m= \pm 1$ denotes the sign of the Doppler shift, $\vec{k}_{0}$

$k_{0}$ is the radar wave vector, and $S(\cdot)$ is the directional ocean wave spectrum, and $\delta(\cdot)$ is the Dirac delta function.

The ratio of spectral power density of two Bragg waves has been previously used to estimate the wind direction [2] and is expressed by

$$
R=\frac{\sigma_{1}\left(\omega_{B}\right)}{\sigma_{1}\left(-\omega_{B}\right)}=\frac{S\left(-2 \vec{k}_{0}\right)}{S\left(2 \vec{k}_{0}\right)}
$$

where the " \pm " sign corresponds to the approaching and receding Bragg waves. Typically, the directional ocean wave spectrum has the form

$$
S(\vec{K})=F(K) g(\varphi)
$$

where $K$ and $\varphi$ are the magnitude and direction of $\vec{K}$ respectively, $F(K)$ represents the non-directional wave spectrum, and the directional spreading function $g(\varphi)$ can be described by the Donelan model[8],

$$
g(\varphi)=0.5 \beta \operatorname{sech}^{2}(\beta \varphi)
$$

where $\beta$ is the spreading parameter with range $[0.1,3]$ in this paper. $\varphi$ is an angle referenced to the mean wave direction. Using the equation (2), (3) and (4), the wind direction $\varphi_{w}$ can be written as

$$
\varphi_{w}=\varphi_{0} \pm \theta
$$

This work was sponsored by the National Marine Technology Program for Public Welfare (no. 201505002) and China Postdoctoral Science Foundation (Grant No. 2013M531559). 
where $\varphi_{0}$ is the radar beam direction, $\theta$ is the angle between the wind direction and the radar beam direction given by

$$
\theta=\frac{1}{2 \beta} \ln \left|\frac{R^{1 / 2} e^{\beta \pi}-1}{1-R^{1 / 2} e^{-\beta \pi}}\right|
$$

To remove this wind direction ambiguity, the "+"or "_" sign in equation (5) should be determined. We have developed ACMB algorithm to eliminate the ambiguity which is applied by comparing the included angles between three adjacent radar beams and the wind direction [6].

In order to retrieve the wind direction, Gurgel proposed a method to find a unique solution by means of least square principle[8]

$$
\begin{aligned}
& \operatorname{LSM}\left(\varphi_{w}\right)=\left[R_{1}-\frac{g\left(\pi+\varphi_{1}-\varphi_{w}\right)}{g\left(\varphi_{1}-\varphi_{w}\right)}\right]^{2} \\
& +\left[R_{2}-\frac{g\left(\pi+\varphi_{2}-\varphi_{w}\right)}{g\left(\varphi_{2}-\varphi_{w}\right)}\right]^{2}
\end{aligned}
$$

where the indices " 1 " and " 2 " refer to the two radar sites and $R_{1}$ and $R_{2}$ represent the ratio of positive and negative Bragg peaks from the same area which is detected by the tow radar. We apply this algorithm to the wind direction elimination of the monostatic radar and the Eq.(7) can be written as

$$
\operatorname{LSM}\left(\varphi_{w}\right)=\sum_{i=1}^{n} \sum_{j=1}^{m}\left[R_{i j}-\frac{g\left(\pi+\varphi_{i}-\varphi_{w}\right)}{g\left(\varphi_{i}-\varphi_{w}\right)}\right]^{2}
$$

where the index $i$ and $j$ represent the $i$ th beam and $j$ th range cell. We can choose nine cells to estimate the direction, i.e. $\mathrm{n}=\mathrm{m}=3$, and $\varphi_{i}$ represents the $i$ th beam direction. Actually the Eq.(8) is one of the calculation way to carry out the multibeam method by least square principle (LSMB).

\section{Data Processing and Analysis with the Algorithms}

In this evaluation of the different algorithms for wind direction we are using data from Fujian station over the period 1-31 October 2013. The radar system OS121H has a work frequency of $7.815 \mathrm{MHz}$, which is developed by CSIC Pengli (Nanjing) Atmosphere and Ocean Information System Co. Ltd. The receiving array of the radar system consists of 24 antennas arranged in two rows of 12 . The specific parameters of this radar system are listed in Table 1. The in-situ data are proved by an anemometer installed on the buoy, which is deployed in the site about $70 \mathrm{~km}$ away the HF transmitter station. In the data processing, we use half-hour means of the radar measurements and the same of the in-situ measurements.
Table 1. The specific parameters of the radar system

\begin{tabular}{|l|l|}
\hline Technical Specifications & value \\
\hline Operating frequency(MHz) & 7.815 \\
\hline Transmitter power $(\mathrm{W})$ & 200 \\
\hline Radial resolution $(\mathrm{km})$ & 5 \\
\hline Azimuthal resolution $\left(^{\circ}\right)$ & 10 \\
\hline
\end{tabular}

The results of the wind direction inverted from the HF radar by ACMB are shown in Fig.1 and the in-situ measurements are also given. From the Fig.1, we can see that most of the wind directions in the in-situ measurements are in the range of $20^{\circ}$ to $50^{\circ}$. The majority of the wind directions inversion from radar is bigger than the in-situ measurements. Fig.2 gives the statistics of wind direction absolute error between in-situ measurements and HF estimates with different algorithms. The figure displays that the results obtained by ACMB are closest to the in-situ data. We get a correlation coefficient of 0.71 and RMS error (RMSE) of $24.8^{\circ}$. And the RMSEs obtained by LSMB and ML are $33.16^{\circ}$ and $42.85^{\circ}$ respectively. Obviously, the RMSE from AMB is less than the other algorithms. The $\beta$ parameter obtained from the ACMB algorithms is different from the LSMB algorithm, which may be one reason for the results of the ACMB and LSMB are different obviously. The RMSE of $24.8^{\circ}$ is low compared the results given by $[6,10,11]$ and close the mean difference $20^{\circ}$ by Huang et al. [4]. Only considering from the aspects of sea state environment, there are two factors resulting in the low RMSE. One is that the varieties of wind directions are slight, and the other is that the wind speeds are high and sustained during the experiment. The distortions of the results calculated by $\mathrm{ML}$ is large than the others, so the RMSE error of $42.85^{\circ}$ is highest among the three cases. Although the correlation coefficient of 0.78 is biggest, the advantage is not obvious compared the others.

The radar operates at $7.815 \mathrm{MHz}$, and using this frequency the phase speed of the ocean wave at the corresponding Bragg frequency $(0.285 \mathrm{~Hz})$ is calculated to be $5.47 \mathrm{~m} / \mathrm{s}$. Hence, we choose $5.47 \mathrm{~m} / \mathrm{s}$ as the cut-off speed of the wind. Then the correlation and RMSE of the data whose wind speeds are less or greater than $5.47 \mathrm{~m} / \mathrm{s}$ are calculated. The results are displayed in Table 2. And the scatter plots of the radar results with the in-situ data are given in the Fig.3. The data whose wind speeds are above and below $5.47 \mathrm{~m} / \mathrm{s}$ are represented by red circle and black plus sign respectively in the figure. During high speed wind conditions, the correlations of the three algorithms increase, while the RMSEs decease. And the differences of RMSE among the three algorithms become little. The accuracy of the results from ACMB improves slight, because the number of data with wind speed $>5.47 \mathrm{~m} / \mathrm{s}$ are much more than the data speed below this value and almost all of the data concentrate near the line of $45^{\circ}$. 


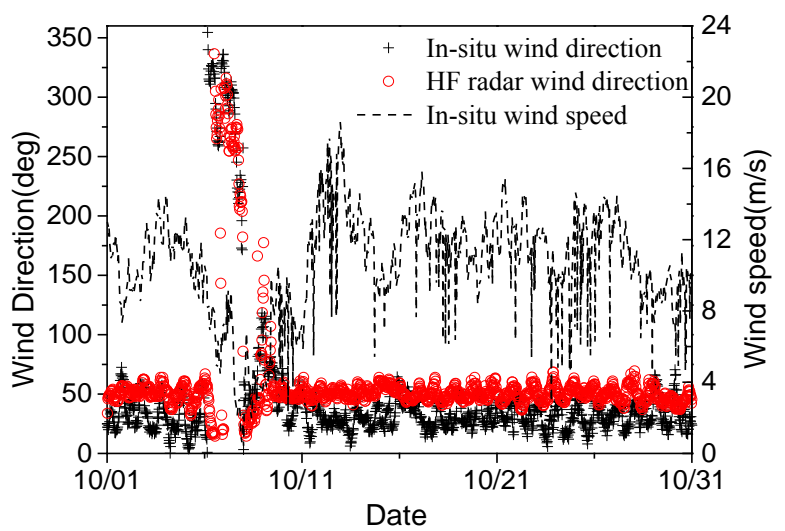

Figure 1. Time series of radar-derived wind directions versus in-situ measurements using ACMB algorithm.

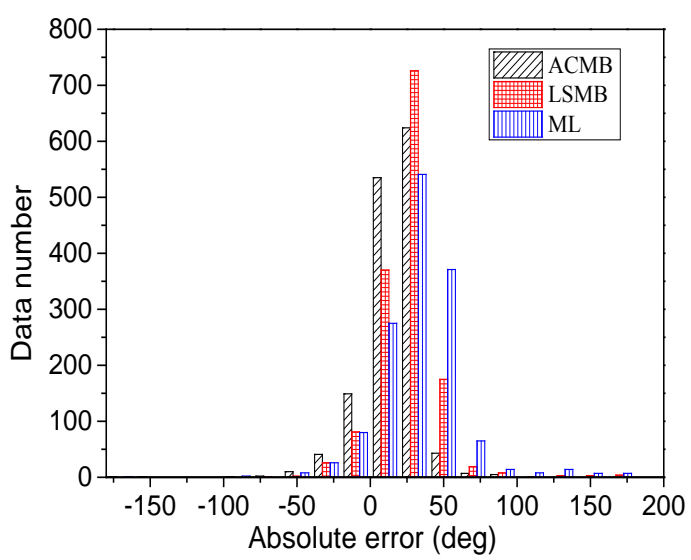

Figure 2. Statistics of wind direction absolute error between HF estimates and in-situ measurements.

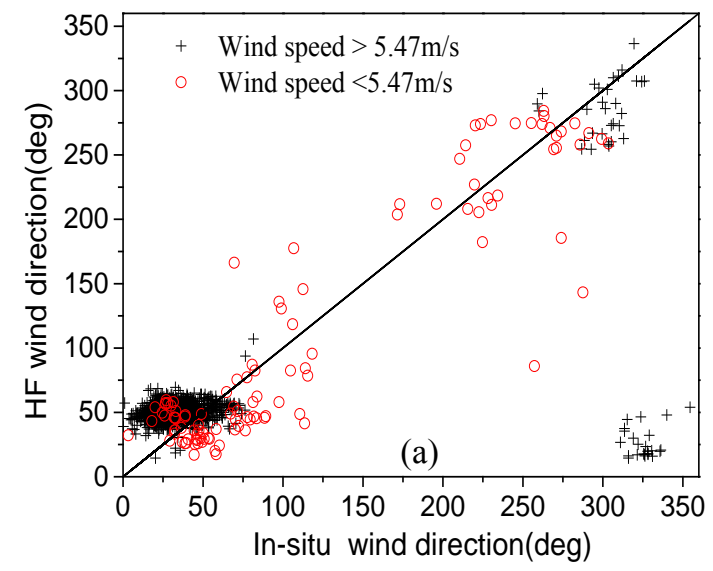

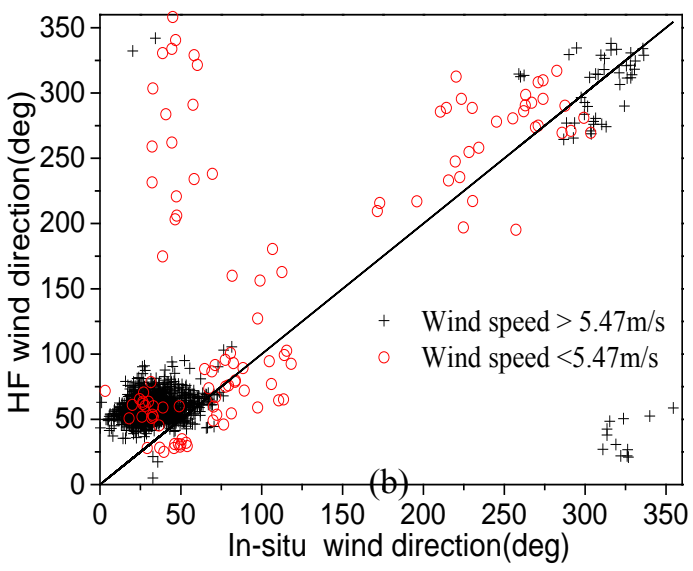

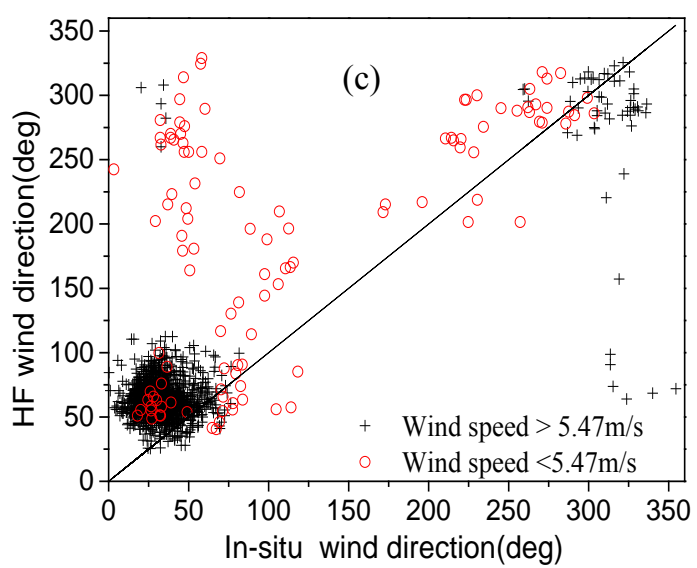

Figure 3. Scatter plots for radar-derived wind directions versus in-situ data. The solid line is at $45^{\circ}$ using (a) ACMB algorithm, (b) LSMB algorithm, and (c) the ML algorithm

Table 2. Results comparison from three algorithm

\begin{tabular}{|c|c|c|c|c|}
\hline $\begin{array}{c}\text { Inversion } \\
\text { algorithm }\end{array}$ & Correlation & $\operatorname{RMSE}\left(^{\circ}\right)$ & $\begin{array}{c}\text { Correlation } \\
(\boldsymbol{U}>5.47 \mathrm{~m} / \mathbf{s})\end{array}$ & $\begin{array}{c}\mathrm{RMSE}\left({ }^{\circ}\right) \\
(\boldsymbol{U}>5.47 \mathrm{~m} / \mathrm{s})\end{array}$ \\
\hline ACMB & 0.71 & 24.80 & 0.72 & 23.52 \\
\hline LSMB & 0.76 & 33.16 & 0.79 & 29.81 \\
\hline ML & 0.78 & 42.85 & 0.82 & 37.67 \\
\hline
\end{tabular}

\section{Discussions}

In this paper, we use three algorithms to extract the wind direction from an experiment data with a monostatic radar system. The results show that all the three algorithms can all eliminate the ambiguity of the wind direction. And the RMSE obtained by the ACMB algorithm is better than the others, which indicates that the ACMB algorithm is best available for extracting wind direction using the data obtained by this experiment. This accuracy of the results is high in that the wind speed is high and sustained. RMSE of the LSMB algorithm and $\mathrm{ML}$ algorithm is closer to the ACMB when data speed above $5.47 \mathrm{~m} / \mathrm{s}$ used. The RMSE obtained by ML is higher than the others in data processing, however, this does not mean that this algorithm is not fit to extract the wind direction from the single HF radar. The ML method has been 
tested in some experiments [3,12]. Further work needs to be done to analyze which algorithm is more available for all kinds of sea state environment by using more experiments data with different conditions.

\section{ACKNOWLEDGMENT}

The authors wish to thank Professor Tao Zhou of CSIC Pengli (Nanjing) Atmosphere and Ocean Information System Co. Ltd. for providing the HF radar $(\mathrm{OS} 121 \mathrm{H})$ and in-situ data.

\section{References}

[1] Barrick, D. E., "Extraction of wave parameters from measured HF seaecho Doppler spectra", Radio Sci., 1977, 12, pp.415-424.

[2] Heron, M. L., Rose, R. J., "On the application of HF ocean radar to the observation of temporal and spatial changes in wind direction", IEEE J. Ocean. Eng., 1986, OE-11(2), pp.210-218.

[3] Wyatt, L. R., Ledgard, L. J. and Anderson, C. W., "Maximum likelihood estimation of the directional distribution of $0.53-\mathrm{Hz}$ Ocean Waves", J. Atmos. Oceanic Technol., 1997, 14(1), pp.591-603.

[4] Huang,W., Gill, E., Wu, S. et al., "Measuring surface wind direction by monostatic HF ground-wave radar at the eastern China sea", IEEE J. Ocean. Eng., 2004, 29(4), pp.1032-1037.

[5] Shen W, "An algorithm to derive wind speed and direction as well as ocean wave directional spectra from $\mathrm{HF}$ radar backscatter measurements based on neural network", Ph.D. dissertation, University of Hamburg, Germany, 2011.

[6] Xiaoliang Chu, Jie Zhang, et al., Algorithm to eliminate the wind direction ambiguity from the monostatic high-frequency radar backscatter spectra, IET Radar, Sonar and Navigation, in press.

[7] Barrick, D., "Remote sensing of sea state by radar", OCEANS Conference-OCEANS, 1972, pp.186-192.
[8] Donelan, M., Hamilton, A. J. and Hui, W. H., "Directional spectra of wind generated waves", Philos.Trans.Roy.Soc.London. A., 1985, 315, pp.509-562.

[9] Gurgel, K. W., Essen, H. H. and Schlick, T., "An empirical method to derive ocean waves from second-order Bragg scattering, prospects and limitations", IEEE J. Ocean. Eng., 2006, 31(4), pp.804-811.

[10] Harlan, J.A. and Georges, T. M., "An empirical relationship between ocean surface wind direction and the Bragg line ratio of sea-echo spectra”, J. Geophys. Res. Ocean., 1994, 99(C4), pp.7971-7978.

[11] Archer, M., "WERA HF radar Measurements of wind direction in the South Atlantic Bight”, B.Sc Ocean Science Dissertation, U. Plymouth., 2008.

[12] Wyatt, L. R., "An evaluation of wave parameters measured using a single HF radar system", Canadian Journal of Remote Sensing, 2002, 28 , pp205-218.

[13] G. Eason, B. Noble, and I.N. Sneddon, "On certain integrals of Lipschitz-Hankel type involving products of Bessel functions," Phil. Trans. Roy. Soc. London, vol. A247, pp. 529-551, April 1955. (references)

[14] J. Clerk Maxwell, A Treatise on Electricity and Magnetism, 3rd ed., vol. 2. Oxford: Clarendon, 1892, pp.68-73.

[15] I.S. Jacobs and C.P. Bean, "Fine particles, thin films and exchange anisotropy," in Magnetism, vol. III, G.T. Rado and H. Suhl, Eds. New York: Academic, 1963, pp. 271-350.

[16] K. Elissa, "Title of paper if known," unpublished.

[17] R. Nicole, "Title of paper with only first word capitalized," J. Name Stand. Abbrev., in press.

[18] Y. Yorozu, M. Hirano, K. Oka, and Y. Tagawa, "Electron spectroscopy studies on magneto-optical media and plastic substrate interface," IEEE Transl. J. Magn. Japan, vol. 2, pp. 740-741, August 1987 [Digests 9th Annual Conf. Magnetics Japan, p. 301, 1982].

[19] M. Young, The Technical Writer's Handbook. Mill Valley, CA: University Science, 1989. 